\title{
Robot-Assisted Free Flap in Head and Neck Reconstruction
}

\author{
Han Gyeol Song, In Sik Yun, Won Jai Lee, Dae Hyun Lew, Dong Kyun Rah \\ Department of Plastic and Reconstructive Surgery, Institute for Human Tissue Restoration, Severance Hospital, Yonsei University College of \\ Medicine, Seoul, Korea
}

Background Robots have allowed head and neck surgeons to extirpate oropharyngeal tumors safely without the need for lip-split incision or mandibulotomy. Using robots in oropharyngeal reconstruction is new but essential for oropharyngeal defects that result from robotic tumor excision. We report our experience with robotic free-flap reconstruction of head and neck defects to exemplify the necessity for robotic reconstruction.

Methods We investigated head and neck cancer patients who underwent ablation surgery and free-flap reconstruction by robot. Between July 1, 2011 and March 31, 2012, 5 cases were performed and patient demographics, location of tumor, pathologic stage, reconstruction methods, flap size, recipient vessel, necessary pedicle length, and operation time were investigated.

Results Among five free-flap reconstructions, four were radial forearm free flaps and one was an anterolateral thigh free-flap. Four flaps used the superior thyroid artery and one flap used a facial artery as the recipient vessel. The average pedicle length was $8.8 \mathrm{~cm}$. Flap insetting and microanastomosis were achieved using a specially manufactured robotic instrument. The total operation time was 1,041.0 minutes (range, 814 to 1,132 minutes), and complications including flap necrosis, hematoma, and wound dehiscence did not occur.

Conclusions This study demonstrates the clinically applicable use of robots in oropharyngeal reconstruction, especially using a free flap. A robot can assist the operator in insetting the flap at a deep portion of the oropharynx without the need to perform a traditional mandibulotomy. Robot-assisted reconstruction may substitute for existing surgical methods and is accepted as the most up-to-date method.

Keywords Robotics / Free tissue flaps / Head and neck neoplasms
Correspondence: Dae Hyun Lew Department of Plastic and Reconstructive Surgery, Institute for Human Tissue Restoration, Severance Hospital, Yonsei University College of Medicine, 250 Seongsan-ro, Seodaemun-gu, Seoul 120-752, Korea

Tel: +82-2-2228-2217

Fax: +82-2-393-6947

E-mail:dhlew@yuhs.ac
This article was presented at the the 2nd Research \& Reconstructive Forum on June 1-2, 2012 in Gwangju, Korea.

No potential conflict of interest relevant to this article was reported.

\section{INTRODUCTION}

Robotic surgical procedures have enhanced the technical capabilities of surgeons with minimally invasive alternatives to traditional surgical procedures in several areas, including urology, gynecology, and general surgery [1]. Recently, the prevalence of robot-assisted head and neck cancer surgery has increased [2]. Robot-assisted radical resection of primary head and neck tumors, known as transoral robotic surgery, enables head and neck surgeons to safely resect oropharyngeal tumor that would have previously been inaccessible without traditional approaches involving a lip-split incision and mandibulotomy. Furthermore,

Copyright $(\odot 2013$ The Korean Society of Plastic and Reconstructive Surgeons

This is an Open Access article distributed under the terms of the Creative Commons Attribution Non-Commercial License (http://creativecommons.org/

licenses/by-nc/3.0/) which permits unrestricted non-commercial use, distribution, and reproduction in any medium, provided the original work is properly cited.

www.e-aps.org 
robotic neck dissection using a retroauricular incision maximizes the advantage of transoral robotic surgery (i.e., minimally invasive surgery).

The primary advantages of this approach compared with traditional open approaches are easier access to the primary lesion, decreased morbidity for patients with a more rapid return to daily life, and improved cosmesis [2]. Another advantage is preventing the complications of radiotherapy because non-union of the mandible or bony necrosis is relatively common after mandibulotomy followed by radiotherapy.

No reports on the long-term follow-up of head and neck robotic surgery have been published, but many papers report the benefits of robotic head and neck surgery in terms of postoperative quality of life, oncologic safety, and the disease-free survival rate [3-5].

Robotic-assisted head and neck cancer surgery represents an alternative method for the management of oropharyngeal tumors but also necessitates the assessment and development of reconstructive approaches using robots. This application of the robot for oropharyngeal reconstruction is new but essential for oropharyngeal defects that result from robotic tumor excision. Here we report our experience with robotic free-flap reconstruction of head and neck defects and discuss the necessity of robotic reconstruction.

\section{METHODS}

\section{Surgical technique}

Under general anesthesia, the patient was placed in the supine position with slight neck extension. Wide excision of the primary tumor and neck dissection were performed by a head and neck surgery team. In cases in which a robot was used to remove the tumor, transoral robotic surgery (TORS) was performed by an established method $[4,6,7]$. After the removal of the primary tumor, if it was a case in which the robot was used to perform the neck dissection, the skin incision for neck dissection was designed at the retroauricular area (Fig. 1) [8]. The incision was made behind the auricle starting from the lower end of the retroauricular sulcus and proceeded upward to the midpoint of the sulcus, and then smoothly angulated downward $0.5 \mathrm{~cm}$ inside the hairline [8]. A specially designed retractor was used for retroauricular neck dissection and reconstruction.

After the head and neck surgeon finished the removal of the primary tumor and neck dissection completely, the plastic surgery team became involved in the operation. A flap was designed by reconstructing the 3-dimensional shape of the defect. The recipient vessels were prepared in the neck space by using a $2.5 \times$ magnification surgical loupe. In addition, a radial forearm free flap and anterolateral thigh flap were elevated by the conventional method.

A robot (Da Vinci S surgical system, Intuitive Surgical, Sunnyvale, CA, USA) was used for flap insetting in the case covering a deep portion of the pharynx. Two needle driver arms to suture and $30^{\circ}$ angled scope to display the operation field were used. Mattress sutures were performed with 3-0 vicryl. After covering the deep portion of the pharynx by robot, the oral cavity could be covered by conventional manual suture.

Microanastomosis was done by the conventional manual method in the preceding 4 cases. When the superior thyroid artery was used as a recipient vessel, the incision with a retroauricular approach was extended slightly inferior, so that microanastomosis could be performed by the conventional method. In the fifth case, robot-assisted microanastomosis was achieved using two black diamond micro forceps and Potts scissors (Fig. 2). These instruments had fine tips and delicate movement for microanastomosis was possible. Thereby, extension of the incision line at the retroauricular area was no longer necessary.

Fig. 1. The demonstration of a retroauricular incision in a cadaver study

(A) Incision design. (B) Retraction for neck dissection by specially designed retractor.
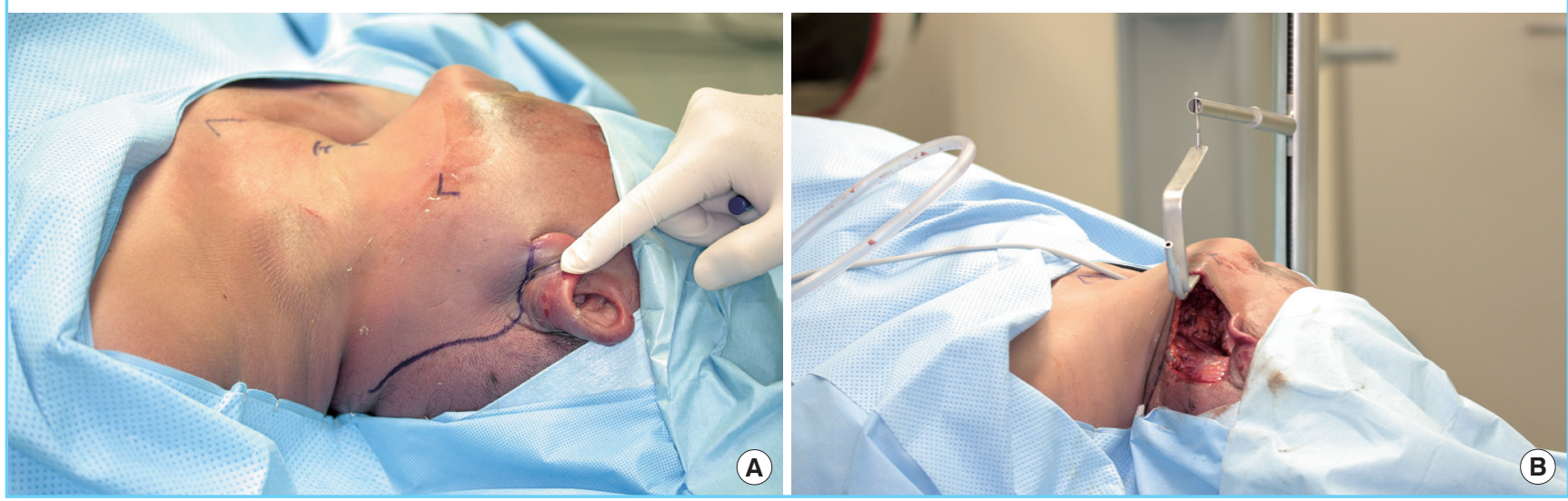


\section{Patient selection}

In this retrospective study, we searched our plastic surgery departmental database to identify all consecutive head and neck cancer patients who underwent robot-assisted operations with the head and neck surgery team and underwent reconstruction with the plastic surgery team between July 1, 2011 and March $31,2012$.

We obtained data on patient demographics, location of tumor, pathologic stage, surgical treatments, flap size, recipient vessel, pedicle length, operation time, and follow-up period from the departmental database and validated these data by using patient medical records.

\section{RESULTS}

Five patients, two males and three females, with a mean age of 55.0 years (range, 46-68 years) were treated for head and neck cancer by minimally invasive robotic surgery between July 1 , 2011 and March 31, 2012. All of the patients had free-flap reconstructions by the plastic surgery department for head and neck defects. Four flaps were radial forearm free flaps, and one flap was an anterolateral thigh free flap (Table 1).

\section{Fig. 2. Instruments for robotic microanastomosis}

(A) Black diamond micro forceps. (B) Potts scissors.
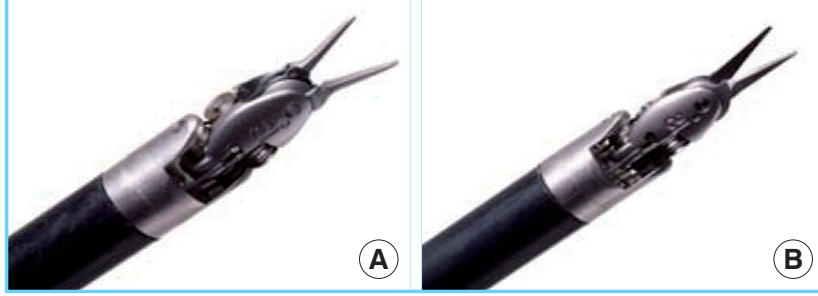

In four patients, the superior thyroid artery was used as a recipient vessel, and the average necessary pedicle length was $9.5 \mathrm{~cm}$. In one patient, the facial artery was used as a recipient vessel, and in this case, the necessary pedicle length was $6.0 \mathrm{~cm}$. The average pedicle length in all cases was $8.8 \mathrm{~cm}$. A branch of the internal jugular vein at the mandible angle area was used as the recipient vein when a retroauricular incision was used for neck dissection. The mean flap size was $56.4 \mathrm{~cm}^{2}$ (range, 28 to $91 \mathrm{~cm}^{2}$ ).

The total operation time was $1,041.0$ minutes (range, 814 to 1,132 minutes), and the time for reconstruction was $591.2 \mathrm{~min}$ utes (range, 536 to 673 minutes). The mean follow up period was 12.8 months (range, 8 to 16 months), and there were no complications including flap necrosis, infection, hematoma, or wound dehiscence in any of the cases during the follow-up period.

Flap insetting was also performed to cover the oral cavity defect using the conventional manual method. However, in cases of a lateral pharyngeal defect after tonsil tumor removal, flap insetting was possible with robot-assisted suturing (Fig. 3).

Microanastomosis was performed in an end-to-end fashion in all 5 cases. Generally, it was achieved by using the traditional manual method. However, in the fifth case, it was achieved using specially manufactured robotic arms. In this case, it was difficult to anastomose the vessel by the traditional method due to a limited ability to visualize the vessel after robot-assisted neck dissection through the retroauricular incision. If we tried to anastomose the vessel manually, it was necessary to extend the neck incision, and it would result in a more noticeable scar. Therefore, we tried to anastomose the vessel by using a robot (Fig. 4). It took about 150 minutes to anastomose the vessel completely. There were no problems including leakage at the anastomosis site, and a milking test for the anastomosed vessel showed strong patency.

\section{Table 1. Patient profile}

\begin{tabular}{|c|c|c|c|c|c|c|c|c|c|c|c|c|}
\hline Case & $\begin{array}{l}\text { Age } \\
(y r) / \\
\text { Sex }\end{array}$ & $\begin{array}{l}\text { Tumor } \\
\text { location }\end{array}$ & $\begin{array}{l}\text { Pathol- } \\
\text { ogy stage } \\
\text { (TNM) }\end{array}$ & $\begin{array}{l}\text { Tumor } \\
\text { op. }\end{array}$ & $\begin{array}{c}\text { Robotic } \\
\text { procedure in } \\
\text { reconstruction }\end{array}$ & $\begin{array}{l}\text { Flap } \\
\text { type }\end{array}$ & $\begin{array}{l}\text { Flap } \\
\text { size } \\
\left(\mathrm{cm}^{2}\right)\end{array}$ & $\begin{array}{l}\text { Recipient } \\
\text { vessel }\end{array}$ & $\begin{array}{l}\text { Pedicle } \\
\text { length } \\
\text { (cm) }\end{array}$ & $\begin{array}{l}\text { Total } \\
\text { op. time } \\
\text { (min) }\end{array}$ & $\begin{array}{l}\text { Reconstruc- } \\
\text { tive op. time } \\
\text { (min) }\end{array}$ & $\begin{array}{l}\text { Follow-up } \\
\qquad(\mathrm{mo})\end{array}$ \\
\hline 1 & $57 / F$ & Tongue & $\begin{array}{l}\text { T1NOM0/ } \\
\text { stage I }\end{array}$ & CWE + RAND & - & RFFF & $8.5 \times 6$ & STA & 9 & 1,012 & 546 & 16 \\
\hline 2 & $46 / F$ & $\begin{array}{l}\text { Base of } \\
\text { tongue }\end{array}$ & $\begin{array}{l}\text { T1NOMO/ } \\
\text { stage I }\end{array}$ & CWE + RAND & - & RFFF & $7 \times 5$ & STA & 9 & 814 & 536 & 15 \\
\hline 3 & $50 / F$ & Tonsil & $\begin{array}{l}\text { T2N2cMO/ } \\
\text { stage IVA }\end{array}$ & TORS + CND & Flap insetting & RFFF & $7 \times 4$ & STA & 10 & 1,126 & 540 & 15 \\
\hline 4 & $68 / \mathrm{M}$ & Tonsil & $\begin{array}{l}\text { T2N1M0/ } \\
\text { stage III }\end{array}$ & TORS + RAND & Flap insetting & $\begin{array}{l}\text { ALT free } \\
\text { flap }\end{array}$ & $13 \times 7$ & STA & 10 & 1,121 & 661 & 10 \\
\hline 5 & $54 / \mathrm{M}$ & Tonsil & $\begin{array}{l}\text { T3NOM0/ } \\
\text { stage III }\end{array}$ & TORS + RAND & $\begin{array}{c}\text { Flap insetting \& } \\
\text { microanastomosis }\end{array}$ & RFFF & $11 \times 7$ & FA & 6 & 1,132 & 673 & 8 \\
\hline Mean & $55 /-$ & - & - & - & - & - & 56.4 & - & 8.8 & $1,041.0$ & 591.2 & 12.8 \\
\hline
\end{tabular}


Fig. 3. Reconstruction after wide excision of left tonsil cancer (case No. 4)

(A) Preoperative image $(13 \mathrm{~cm} \times 7 \mathrm{~cm}$ defect in the soft palate, left tonsil, and lateral pharyngeal wall). (B) After anterolateral thigh flap elevation (flap size, $13 \mathrm{~cm} \times 7 \mathrm{~cm}$ ). (C) Microanastomosis of superior thyroid artery to descending branch of lateral femoral circumflex artery by retroauricular incision. (D) Robotic flap insetting with two needle driver arms.
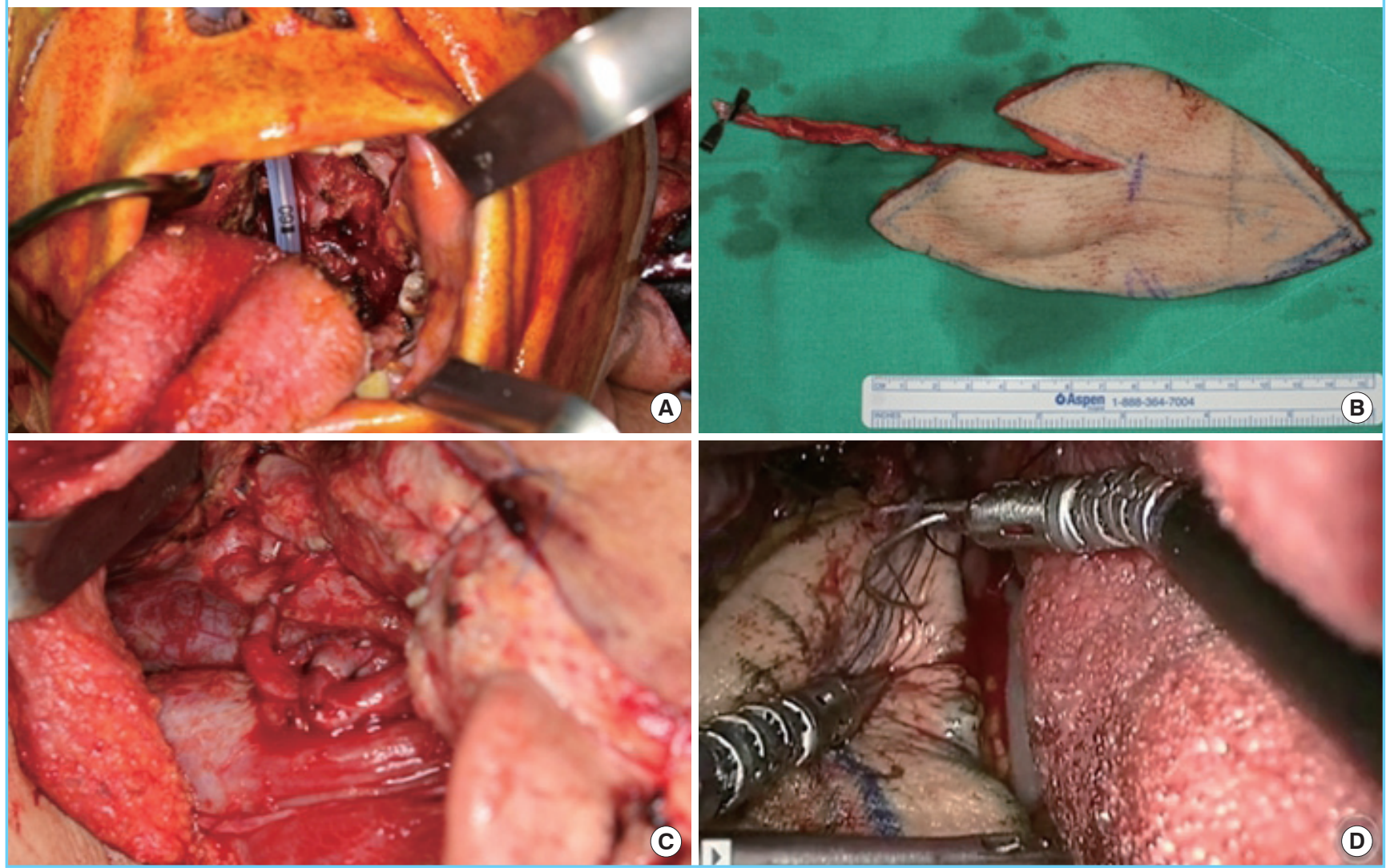

Fig. 4. Microanastomosis by specially manufactured robotic arm

(A) Artery anastomosis by two black diamond micro forceps and Potts scissors. (B) Vein anastomosis by the same method.

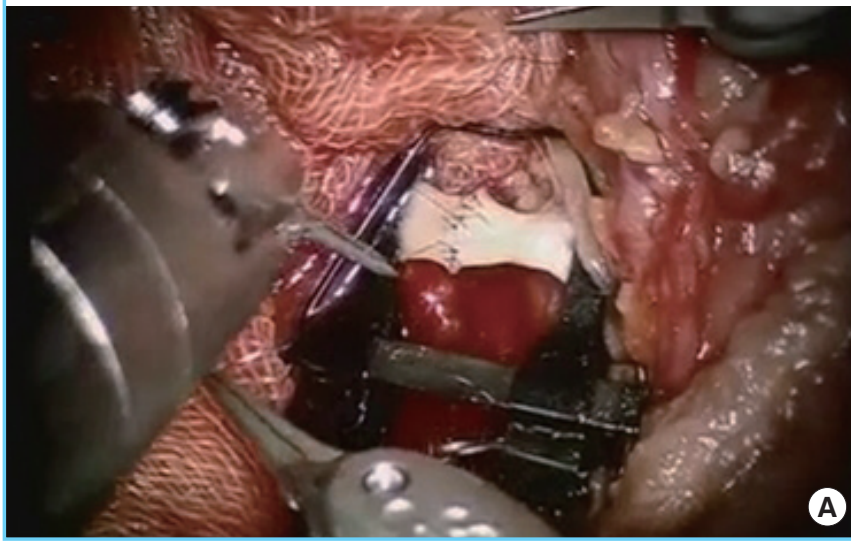

\section{DISCUSSION}

Since its introduction in the mid-1990s, the use of robotic surgery has increased in various fields. In addition, robotic procedures in head and neck surgery have recently been performed. The areas in head and neck surgery in which robots are used are

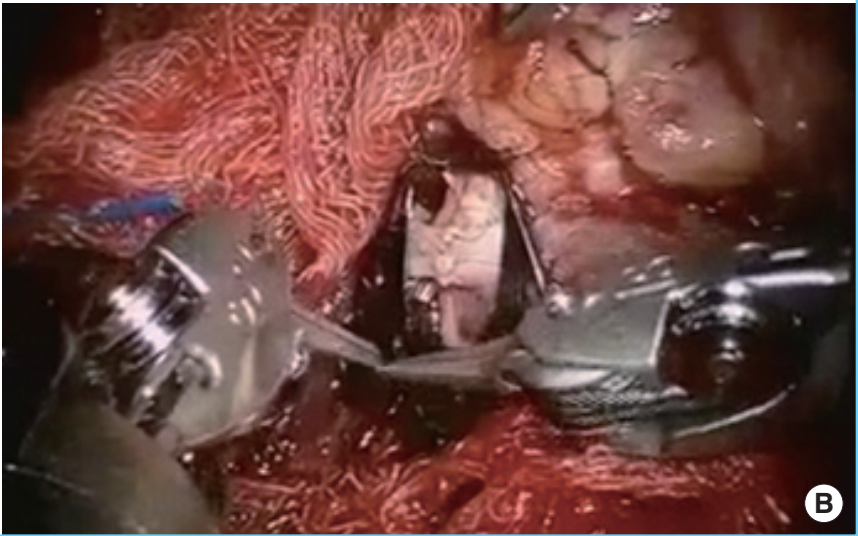

TORS and robotic neck dissection. Robot-assisted head and neck surgery is at issue in the otolaryngology section [7].

In the past, AlloDerm (LifeCell Corp., Branchburg, NJ, USA) grafts were commonly used to cover defects because the indications for TORS and robot-assisted neck dissection through a retroauricular incision were limited. Only early-stage malignan- 
cies were an indication for robotic surgery. In these cases, there was no connection between the oropharyngeal defect and neck space, and the major vessel in the neck was not exposed. Consequently, repair of oropharyngeal defects by robotic surgery was simple and relatively small.

Indications for TORS and robotic neck dissection have gradually been expanded, including larger and more complex defects that cannot be covered with a simple AlloDerm graft. If the carotid artery was exposed in the neck space, or a soft tissue tunnel was present between the pharynx and neck space, the complex defect should be covered by local tissue transfer or free tissue transfer. In addition, plastic surgeons have become more skilled in handling robots and are more likely to attempt flap inset and microanastomosis by robot-assisted methods.

Transoral robotic surgery, which safely extirpates head and neck tumors without the mandible swing approach, and roboticassisted neck dissection using a retroauricular approach, which makes the incisional scar less noticeable, are considered minimally invasive surgery. However, these minimally invasive incisions are problematic for plastic and reconstructive surgery because the space available to perform the reconstructive surgery may be restricted. With advances in transoral robotic surgery, larger defects pose challenges for the reconstructive surgeon. Preserving a competent velopharyngeal sphincter, a watertight seal between the pharynx and neck, and adequate sensation and volume in the tongue base are necessary to optimize the physiological function of the oropharynx and minimize functional deficits.

Robotic reconstruction is a solution to these problems. The robot's precision and improved visualization in confined spaces makes it more appropriate for reconstruction in TORS and robotic neck dissection. In other words, robot-assisted flap insetting and microanastomosis are needed to reconstruct the oropharyngeal defect resulting from TORS and robotic neck dissection. The robotic inset of flap or microvascular anastomosis allows anatomically confined regions to be accessed and reconstructed by providing a high-resolution, high-magnification image and precise instrumentation [9].

Park et al. [10] reported the treatment outcomes by robotassisted neck dissection. In this report, the robot-assisted neck dissection group had many advantages, such as less noticeable scars and a good wound healing process. However, in terms of comparison of operation time between the robot-assisted neck dissection group and open neck dissection group, the robot-assisted neck dissection group showed a significantly longer operation time than the open neck dissection group. In our study, the robot-assisted reconstruction group indicated a similarly longer operation time than the conventional reconstruction group after robotic head and neck surgery. One of the reasons for this delay was that it takes a long time to set the robotic arm. One way to solve this problem may be to conduct the parts of surgery requiring the robot all at once, not separately, as it is quite time consuming to alternate between operating robotically and manually. Therefore, after robot-assisted removal of the primary tumor and neck dissection, the plastic surgery team performed robotic microanastomosis and flap insetting immediately. To do so, flap elevation and tumor removal at the same time, in other words, a two team approach, could be one solution. Another reason is that there is a long learning curve period for the plastic surgeon to become acquainted with using the robot.

Robotic microvascular anastomosis is a growing application. In neck dissection by retroauricular incision, it is more difficult to find an appropriate recipient vessel than with conventional open neck dissection because of limited visualization. In addition, it is more difficult to perform microanastomosis of the vessel through the narrow space using a conventional microscope. Notwithstanding, the greatest difficulty faced in operating by conventional methods of neck dissection by retroauricular incision is visualization of the vessels in an oblique view, at an approximately $45^{\circ}$ angle, not upright from the vessel at a $90^{\circ}$ angle. Furthermore, conventional methods necessitate longer dissection of the recipient vessel and require the vessel to be pulled to the incision site for anastomosis. Additionally, excessive traction to see the vessel in conventional methods may harm the retroauricular skin flap margin.

Therefore, robotic-assisted microanastomosis is an alternative method in cases of narrow and limited operative fields after robotic head and neck surgery. The robot provides superior visualization, access, and precision in areas that can only be approached with great technical difficulty under normal conditions. The superior access the robot affords is what drives the movement among plastic surgeons to use the robot for oropharyngeal reconstruction.

It was common to use the superior thyroid artery and vein as recipient vessels in traditional open surgery. With retroauricular robotic neck dissection, the superior thyroid vessel was located inferior to the incision site, making it difficult to use as the recipient vessel. However, the facial artery and branch of the internal jugular vein at the angle of the mandible were used easily. The surgeon performing the TORS should be warned so that the facial artery and branch of the jugular vein at the angle of the mandible are not sacrificed during neck dissection surgery, if possible.

For performing robotic surgery, three points must be considered. First, special instruments such as black diamond micro forceps and Potts scissors should be prepared. However, more 
delicate instruments should be developed for robot-assisted surgery because, as of yet, there is no haptic feedback to the operator in robotic surgery. Second, there was the additional cost to the patient for robotic surgery compared to the conventional procedure. Because robotic surgery has many advantages such as enhanced cosmesis, postoperative quality of life, oncologic safety, and disease-free survival rate [2-5], it could be an additional option for patients. Third, it is necessary to evaluate the long-term outcomes of the operation. To date, superior esthetic and functional outcomes have been reported after reconstruction surgery following robotic head and neck surgery. However, further research should be performed to improve reconstruction methods.

Minimally invasive resections are of great benefit to patients and represent a true advance in the field of head and neck surgery. If such techniques are to become standard, reconstructive methods must keep pace. This study demonstrates the clinically applicable use of robots in oropharyngeal reconstruction, especially using a free flap. In this study, no complications occurred in robot-assisted microanastomosis and flap insetting. Robots can assist the operator in insetting the flap in a deep portion of the oropharynx without the need to perform a traditional mandibulotomy. Robot-assisted reconstruction may substitute for existing methods of surgery and is accepted as the most up-todate method.

\section{REFERENCES}

1. Selber JC, Baumann DP, Holsinger FC. Robotic latissimus dorsi muscle harvest: a case series. Plast Reconstr Surg 2012; 129:1305-12.
2. Aubry K, Yachine M, Perez AF, et al. Transoral robotic surgery for head and neck cancer: a series of 17 cases. Eur Ann Otorhinolaryngol Head Neck Dis 2011;128:290-6.

3. Park YM, Kim WS, Byeon HK, et al. Oncological and functional outcomes of transoral robotic surgery for oropharyngeal cancer. Br J Oral Maxillofac Surg 2013;51:408-12.

4. Park YM, Kim WS, De Virgilio A, et al. Transoral robotic surgery for hypopharyngeal squamous cell carcinoma: 3-year oncologic and functional analysis. Oral Oncol 2012;48:560-6.

5. Hurtuk AM, Marcinow A, Agrawal A, et al. Quality-of-life outcomes in transoral robotic surgery. Otolaryngol Head Neck Surg 2012;146:68-73.

6. Genden EM, Desai S, Sung CK. Transoral robotic surgery for the management of head and neck cancer: a preliminary experience. Head Neck 2009;31:283-9.

7. Kim WS, Lee HS, Kang SM, et al. Feasibility of robot-assisted neck dissections via a transaxillary and retroauricular ("TARA") approach in head and neck cancer: preliminary results. Ann Surg Oncol 2012;19:1009-17.

8. Lee HS, Kim WS, Hong HJ, et al. Robot-assisted Supraomohyoid neck dissection via a modified face-lift or retroauricular approach in early-stage cN0 squamous cell carcinoma of the oral cavity: a comparative study with conventional technique. Ann Surg Oncol 2012;19:3871-8.

9. Selber JC. Transoral robotic reconstruction of oropharyngeal defects: a case series. Plast Reconstr Surg 2010;126:1978-87.

10. Park YM, Holsinger FC, Kim WS, et al. Robot-assisted selective neck dissection of levels II to V via a modified facelift or retroauricular approach. Otolaryngol Head Neck Surg 2013;148:778-85. 